\title{
On the Unique Solvability of the Generalized Absolute Value Matrix Equation
}

\author{
Kai Xie \\ College of Mathematics and Statistics, Northwest Normal University, Lanzhou, P. R. China \\ Email address: \\ XKK740418XKK@163.com \\ To cite this article: \\ Kai Xie. On the Unique Solvability of the Generalized Absolute Value Matrix Equation. American Journal of Applied Mathematics. \\ Vol. 9, No. 4, 2021, pp. 104-107. doi: 10.11648/j.ajam.20210904.12
}

Received: July 13, 2021; Accepted: July 26, 2021; Published: August 2, 2021

\begin{abstract}
The generalized absolute value matrix equation has application in a variety of optimization problems, its unique solvability is still on the way. In this note, the unique solvability of the generalized absolute value matrix equation is considered. A new unique solvability of generalized absolute value matrix equation is given. The obtained result can be regarded as an extension of the absolute value equation to the generalized absolute value matrix equation. As an application, new convergence of matrix multisplitting Picard-iterative method is presented.
\end{abstract}

Keywords: Generalized Absolute Value Matrix Equation, Unique Solution, Singular Values

\section{Introduction}

In this note, the following generalized absolute value matrix equation (GAVME) is considered

$$
A X+B|X|=C
$$

where $A, B, C \in C^{\mathrm{n} \times \mathrm{n}}$ are given matrices and $X \in C^{\mathrm{n} \times \mathrm{n}}$ is an unknown matrix, $|X|$ denotes the component-wise absolute value of the matrix $X$, i.e., $|X|=\left(\left|x_{i j}\right|\right)$. The GAVME (1) is a generalization of the generalized absolute value equation (GAVE)

$$
A x+B|x|=c,
$$

where $A, B \in C^{\mathrm{n} \times \mathrm{n}}$ are given matrices, $c \in C^{\mathrm{n}}$ is given vector and $\mathrm{x} \in C^{\mathrm{n}}$ is an unknown vector. The GAVE (2) was introduced by Rohn [1] and investigated in a more general context [2-4]. The GAVME (1) is also a generalization of the absolute value equation (AVE)

$$
A x+|x|=c .
$$

The nonlinear and non-differentiable term $B|X|$ in (1) often leads to more challenges of the unique solvability and numerical methods for (1) compared with matrix equations, there are many results of solvability and numerical methods for some kinds of matrix equations, see for example [5-8] and references cited therein.
The GAVE (2) and AVE (3) have recently attracted the attention of many scholars because their applications in a variety of optimization problems, e.g., linear complementarity problem, linear programming or convex quadratic programming problems; see for example [1-4, 9]. The conditions of the unique solvability of GAVE (2) and AVE (3) have been given in references [1, 3, 10-13], and the numerical methods for solving GAVE (2) and AVE (3) can be found in the literatures [12-21]. Rohn [22] has reported the condition for (2) for having unique solution. Let $\sigma_{\max }(\cdot)$ and $\sigma_{\min }(\cdot)$ denote the largest and smallest singular value of a given matrix respectively, it is proved in Theorem 2 of [22] that if $\sigma_{\max }(|B|)<\sigma_{\min }(A)$, then for each $c \in C^{\mathrm{n}}$, the equation (2) has a unique solution. Authors of [11] were able to reduce the conditions for having unique solution for (2) and sowed that the solution is unique under the condition

$$
\sigma_{\max }(B)<\sigma_{\min }(A) .
$$

Compared with the GAVE (2) and AVE (3), the GAVME (1) is rarely studied. It is understood that only Dehghan and Shirilord [23] have provided the condition of unique solvability for the GAVME (1) and proposed the matrix multisplitting Picard-iterative method for solving (1) recently. Dehghan and Shirilor [23] have shown that the condition for having uniqueness solution of (1) is the same as that for (2), which is reported by Rohn [22]. In this note, it will be pointed out that the unique solvability of the GAVME (1) in [23] may 
be invalid to judge the unique solution of GAVME in some case, and give a new unique solvability for the GAVME (1), which is weaker than that in [23], and is same as that for GAVE (2) in [11]. The new unique solvability for the GAVME (1) obtained in this note can be regarded as an extension of the GAVE (2) (see [11]) to the GAVME (1). As an application of new result, the convergence of matrix multisplitting Picard-iterative method for solving (1) is restated.

\section{Main Result}

In this section, main result on the unique solvability of the GAVME (1) will be given. First of all, recall the result about the unique solvability of the GAVME (1) provided in [23] reads

Lemma 1 ([23] Theorem 2.1) Let $A, B \in C^{n \times n}$ satisfy

$$
\sigma_{\max }(|B|)<\sigma_{\min }(A),
$$

then the GAVME (1) has a unique solution for any matrix $C \in C^{n \times n}$.

It should be remarked that the condition (4) in Lemma 1 may be invalid to judge the unique solution of GAVME in some cases, consider the following simple example.

Example 1 Consider the GAVME

$$
\begin{gathered}
\underbrace{\left[\begin{array}{cc}
2.5 & 0 \\
0 & 2.5
\end{array}\right]}_{A} \underbrace{\left[\begin{array}{ll}
x_{11} & x_{12} \\
x_{21} & x_{22}
\end{array}\right]}_{X}+\underbrace{\left[\begin{array}{cc}
0.5 & 1 \\
-1 & 1 \\
-1 & 1.5
\end{array}\right]}_{B} . \\
\underbrace{}_{C}
\end{gathered}
$$

It is easy to see that $\sigma_{\min }(A)=2.5$ and $\sigma_{\max }(|B|)=$ 2.6180, so $\sigma_{\max }(|B|)<\sigma_{\min }(A)$ does not hold. However, the GAVME (5) is uniquely solvable. In fact, the unique solution of (5) is $X=\left[\begin{array}{ll}1 & 0 \\ 0 & 1\end{array}\right]$.

To give main result, the following result is needed.

Lemma 2 ([11] Theorem 2.1) Let $A, B \in C^{n \times n}$ satisfy

$$
\sigma_{\max }(B)<\sigma_{\min }(A),
$$

then the GAVE (2) has a unique solution for any $c \in C^{n}$.

Theorem 1 Let $A, B \in C^{n \times n}$ satisfy

$$
\sigma_{\max }(B)<\sigma_{\min }(A),
$$

then the GAVME (1) has a unique solution for any matrix $C \in C^{n \times n}$.

Proof. Let $X$ and $C$ be partitioned as $X=\left(x_{1}, \cdots, x_{n}\right)$ and $C=\left(c_{1}, \cdots, c_{n}\right)$, where $x_{j}$ and $c_{j}$ are the $j$-th column of matrices $X$ and $C$, respectively. The fact of $|X|=$ $\left(\left|x_{1}\right|, \cdots,\left|x_{n}\right|\right)$ implies that the GAVME (1) can be rewritten as

$$
A\left(x_{1}, \cdots, x_{n}\right)+B\left(\left|x_{1}\right|, \cdots,\left|x_{n}\right|\right)=\left(c_{1}, \cdots, c_{n}\right),
$$

or equivalently,

$$
A x_{j}+B\left|x_{j}\right|=c_{j}, j=1,2, \cdots, n .
$$

It follows from Lemma 2 (or Theorem 2.1 of [11]) that the GAVE (7) has a unique solution for any $j \in\{1,2, \cdots, n\}$ when $\sigma_{\max }(B)<\sigma_{\min }(A)$. As a result, the unknown matrix $X=\left(x_{1}, \cdots, x_{n}\right)$ can be unique identified by solving (7), that is to say the GAVME (1) has a unique solution for any matrix $C \in C^{n \times n}$. The proof is completed.

Remark 1 As for $B \in C^{n \times n}, \sigma_{\max }(B) \leq \sigma_{\max }(|B|)$ holds (see Remark 2.1 of [11]), so the condition $\sigma_{\max }(B)<$ $\sigma_{\min }(A)$ is more weaker than $\sigma_{\max }(|B|)<\sigma_{\min }(A)$.

Remark 2 Consider Example 1 again, it can be seen that $\sigma_{\max }(B)=2.3028$ and $\sigma_{\max }(B)<\sigma_{\min }(A)$ holds, so it follows from Theorem 1 that the GAVME (5) has the unique solution. This shows that Theorem 1 is valid in judging the unique solvability of the GAVME (1) while Theorem 2.1 in [23] is invalid.

In particular, when $B=I$, an n-order identity matrix, the GAVME (1) becomes the absolute value matrix equation (AVME)

$$
A X+|X|=C
$$

From Theorem 1, the following unique solvability of the AVME (8) can be derived directly.

Corollary 1 If the singular values of $A \in C^{n \times n}$ outstrip 1, then the AVME (8) has a unique solution for any matrix $C \in C^{n \times n}$.

Remark 3 Corollary 1 can be regarded as extending the unique solvability of the AVE (3) (see Proposition 3 (i) in [3]) to the AVME (8), and Theorem 1 extends the unique solvability of Lemma 2 from the GAVE (2) to the GAVME (1).

\section{An Application}

In this section, as an application of result in the previous section, the convergence of matrix multisplitting Picard-iterative method [23] for solving (1) will be restated. Let $A=M_{j}-N_{j}, j=1,2, \cdots p$, be different splittings for matrix $A$, then the matrix multisplitting Picard-iterative method is defined as [23]:

Method 1 Suppose that $X^{(0)} \in C^{n \times n}$ is an initial guess for solution of the GAVME (1), and $A=M_{j}-N_{j}, j=1,2, \cdots p$, are different splittings for matrix $A$, compute $X^{(k)} \in C^{n \times n}$ using the following iteration:

1. For $k=0,1,2, \cdots$,

2. Set $X^{(k, 0)}=X^{(k)}$,

3 . For $t=0,1, \cdots, t_{k}-1$,

4. Solve the following equations by an iterative method to obtain $X^{(k, t+1)}$,

$$
\left\{\begin{array}{c}
M_{1} X^{(k, t+1 / p)}=N_{1} X^{(k, t)}-B\left|X^{(k)}\right|+C, \\
M_{2} X^{(k, t+2 / p)}=N_{2} X^{(k, t+1 / p)}-B\left|X^{(k)}\right|+C, \\
\vdots \\
M_{p} X^{(k, t+1)}=N_{p} X^{(k, t+1-1 / p)}-B\left|X^{(k)}\right|+C, \\
k=0,1, \cdots, t=0,1, \cdots, t_{k}-1,
\end{array}\right.
$$

5. Set $\boldsymbol{X}^{(\boldsymbol{k}+1)}=\boldsymbol{X}^{\left(t, t_{k}\right)}$

In concise form, Method 1 can be rewritten as 


$$
X^{(k+1)}=\Theta_{p}^{t_{k}}\left(X^{(k)}+A^{-1}\left(\mathrm{~B}\left|X^{(k)}\right|-\mathrm{C}\right)\right)+A^{-1}\left(\mathrm{C}-B\left|X^{(k)}\right|\right),
$$

where $\Theta_{p}=\Pi_{j=1}^{p} M_{j}^{-1} N_{j}$. On the convergence of Method 1, the following result is obtained.

Theorem 2 Let $A, B \in C^{n \times n}$ satisfy $n \sigma_{\max }(B)<$ $\sigma_{\min }(A)$. Suppose that $X^{(0)} \in C^{n \times n}$ is a preliminary guess for solution of the GAVME (1), and $X^{(*)}$ is its exact solution, $\left\{X^{(k)}\right\}_{k=0}^{\infty}$ is generated by Method 1. If $\left\|\Theta_{p}\right\|_{F}<1$, then

$$
\begin{gathered}
\left\|X^{(k+1)}-X^{(*)}\right\|_{F} \\
\leq\left(\left\|\Theta_{p}\right\|_{F}^{\theta}\left(1+\left\|A^{-1}\right\|_{F}\|B\|_{F}\right)\right. \\
\left.+\left\|A^{-1}\right\|_{F}\|B\|_{F}\right)^{k+1}\left\|X^{(0)}-X^{(*)}\right\|_{F}
\end{gathered}
$$

holds, where $\theta=\min _{k}\left\{t_{k}\right\}$. In particular, if

$$
\theta>\frac{\log \left[1-\left\|A^{-1}\right\|_{F}\|B\|_{F}\right]-\log \left[1+\left\|A^{-1}\right\|_{F}\|B\|_{F}\right]}{\log \left(\left\|\Theta_{p}\right\|_{F}\right)}
$$

then the sequence $\left\{X^{(k)}\right\}_{k=0}^{\infty}$ converges to the unique solution $X^{(*)}$.

Remark 4 The difference between Theorem 3.2 of [23] and Theorem 2 is that $n \sigma_{\max }(|B|)<\sigma_{\min }(A)$ is replaced by $n \sigma_{\max }(B)<\sigma_{\min }(A)$. In fact, when $n>1$, there is

$\sigma_{\max }(B)<\sqrt{n} \sigma_{\max }(B)<n \sigma_{\max }(B)$.

Therefore, on the one hand, $n \sigma_{\max }(B)<\sigma_{\min }(A)$ implies $\sigma_{\max }(B)<\sigma_{\min }(A)$, it follows from Theorem 1 that the GAVME (1) has the unique solution for any matrix $C \in C^{n \times n}$, on the other hand, it is easy to see that

$$
\left\|A^{-1}\right\|_{F}\|B\|_{F} \leq n \frac{\sigma_{\max }(B)}{\sigma_{\min }(A)}<1
$$

When $n \sigma_{\max }(B)<\sigma_{\min }(A)$, so $1-\left\|A^{-1}\right\|_{F}\|B\|_{F}>0$ and the conclusion of Theorem 2 holds, see [23].

Remark 5 When $p=1$, Method 1 reduces to Algorithm 1 of [23], hence, the convergence of this case from Theorem 2 can be given directly. The difference is that the condition in Theorem 2.3 of [23] is replaced by $\sigma_{\max }(B)<\sigma_{\min }(A)$.

Remark 6 It should also be remarked that the condition $n \sigma_{\max }(B)<\sigma_{\min }(A)$ given in Theorem 2 is weaker than $n \sigma_{\max }(|B|)<\sigma_{\min }(A)$ in Theorem 3.2 of [23] as $\sigma_{\max }(B) \leq \sigma_{\max }(|B|) \quad($ see Remark 2.1 of $[11])$.

\section{Conclusions}

In this paper, the unique solvability of generalized absolute value matrix equation is discussed. A weaker condition for the unique solvability for the GAVME (1) is given. In addition, as an application of the obtained result, the convergence of matrix multisplitting Picard-iterative method is also restated.

\section{Acknowledgements}

The author is very grateful to anonymous reviewers for their constructive suggestions and careful reading on improving the quality of paper.

The author would like to thank Professor Shu-Xin Miao for his valuable comments and helpful suggestions.

This research was partially supported by the National Natural Science Foundation of China under Grant No. 61967014.

\section{References}

[1] Rohn, J. (2004) A theorem of the alternatives for the equation $\mathrm{Ax}+\mathrm{B}|\mathrm{x}|=\mathrm{b}$. Linear and Multilinear Algebra, 52, 421-426.

[2] Mangasarian, O. L. (2012) Primal-dual bilinear programming solution of the absolute value equation. Optim. Lett., 6, $1527-1533$.

[3] Mangasarian, O. L., \& Meyer, R. R. (2006) Absolute value equations. Linear Algebra Appl., 419, 359-367.

[4] Prokopyev, O. (2009) On equivalent reformulations for absolute value equations. Comput. Optim. Appl., 44, 363-372.

[5] Dehghan, M., \& Hajarian, M. (2008) An iterative algorithm for the reflexive solutions of the generalized coupled Sylvester matrix equations and its optimal approximation. Appl. Math. Comput., 202, 571-588.

[6] Dehghan, M., \& Hajarian, M. (2010) The general coupled matrix equations over generalized bisymmetric matrices. Linear Algebra Appl., 432, 1531-1552.

[7] Dehghan, M., \& Shirilord, A. (2019) A generalized modified Hermitian and skew--Hermitian splitting (GMHSS) method for solving complex Sylvester matrix equation. Appl. Math. Comput., 348, 632-651.

[8] Dehghan, M., \& Shirilord, A. HSS-like method for solving complex nonlinear Yang-Baxter matrix equation. Eng. Comput-Germany. (in press).

[9] Hu, S. L., \& Huang, Z. H. (2010) A note on absolute value equations. Optim. Lett., 4, 417-424.

[10] Wu, S. L., \& Guo, P. (2016) On the unique solvability of the absolute value equation. J. Optim. Theory Appl., 169, 705-712.

[11] Wu, S. L., \& Li, C. X. (2020) A note on unique solvability of the absolute value equation. Optim. Lett., 14, 1957-1960.

[12] Rohn, J., Hooshyarbakhsh, V., \& Farhadsefat, R. (2014) An iterative method for solving absolute value equations and sufficient conditions for unique solvability. Optim. Lett., 8, $35-44$.

[13] Zhang, C., \& Wei, Q. (2009) Global and finite convergence of a generalized newton method for absolute value equations. J. Optim. Theory Appl., 143, 391-403.

[14] Caccetta, L., Qu, B., \& Zhou, G. (2011) A globally and quadratically convergent method for absolute value equations. Comput. Optim. Appl., 48, 45-58.

[15] Gu, X. M., Huang, T. Z., \& Li, H. B., etc. (2017) Two CSCS-based iteration methods for solving absolute value equations. J. Appl. Anal Comput., 7, 1336-1356.

[16] Li, C. X. (2016) A modified generalized Newton method for absolute value equations. J. Optim. Theory Appl., 170, 1055-1059. 
[17] Mangasarian, O. L. (2009) A generalized Newton method for absolute value equations. Optim. Lett., 3, 101-108.

[18] Mangasarian, O. L. (2009) Knapsack feasibility as an absolute value equation solvable by successive linear programming. Optim. Lett., 3, 161-170.

[19] Noor, M. A., Iqbal, J., \& Noor, K. I., etc. (2012) On an iterative method for solving absolute value equations. Optim. Lett., 6, 1027-1033.

[20] Salkuyeh, D. K. (2014) The Picard-HSS iteration method for absolute value equations. Optim. Lett., 8, 2191-2202.
[21] Tang, J., \& Zhou, J. (2019) A quadratically convergent descent method for the absolute value equation $\mathrm{Ax}+\mathrm{B}|\mathrm{x}|=\mathrm{b}$. Oper. Res. Lett., 47, 229-234.

[22] Rohn, J. (2009) On unique solvability of the absolute value equation. Optim. Lett., 3, 603-606.

[23] Dehghan, M., \& Shirilord, A. (2020) Matrix multisplitting Picard-iterative method for solving generalized absolute value matrix euqation. Appl. Numer. Math., 158, 425-438. 